\title{
Symptom Concerns and Resource Utilization in Patients with Lung Cancer
}

\author{
YALE D. PODNOS, M.D., M.P.H., ${ }^{1}$ TAMI R. BORNEMAN, R.N., M.S.N., C.N.S., ${ }^{2}$ \\ MARIANNA KOCZYWAS, M.D., ${ }^{3}$ GWEN UMAN, Ph.D., ${ }^{4}$ \\ and BETTY R. FERRELL, Ph.D., F.A.A.N. ${ }^{2}$
}

\begin{abstract}
Objective: Lung cancer remains a major source of death in the United States. With the aging of the population, health policy makers are challenged to develop systems of care for the complex needs of these patients. This study sought to determine quality of life and symptom concerns in lung cancer patients. The study also sought to determine how supportive care resources were being used to address these concerns.

Methods: One hundred consecutive patients with newly diagnosed lung cancer presenting over a 12-month period were selected from the tumor registry. Charts were reviewed for demographic data, treatment history, treatment received, number and type of practitioner encounters, readmissions, and complications for a 6-month period.

Results: Of the 100 charts retrospectively reviewed, 4 patients had small cell and 96 patients had non-small cell lung cancer. The median age was 67 years. Fifty-three patients were men. The most common symptoms were pain, cough, dyspnea, and fatigue. A total of 114 referrals in 57 patients were made. Nutrition consultation was the most common.

Conclusions: This study serves to guide the institution in the development of more effective support services for patients with lung cancer to address quality of life concerns through collaboration between clinicians and researchers.
\end{abstract}

\section{INTRODUCTION}

$\mathbf{L}$ UNG CANCER is the second most common cancer in the United States, afflicting an estimated 170,000 people in 2006 and resulting in almost 162,000 deaths. ${ }^{1}$ This cancer population is comprised primarily of non-small cell lung cancer and small cell lung cancer. Non-small cell lung cancer is much more frequent, accounting for up to $85 \%$ of cases. ${ }^{2}$ Although arising in the same organ, treatment of these disparate pathologies is largely different. However, their pre- sentations, symptoms, and clinical courses can be quite similar.

Despite recent advances in treatment of these complicated patients, the prognosis remains poor with 5year relative survival rates between $13 \%$ and $16 \%$. $^{3}$ This is especially true in the majority of patients who are diagnosed in the later stages of the disease when curative operations are no longer feasible. As patients progress in their disease, the expected survival drastically decreases while the symptom burden increases. Complicating matters is the relatively advanced age of

\footnotetext{
${ }^{1}$ Department of Surgery, Duke University, Raleigh, North Carolina.

${ }^{2}$ Department of Nursing Education and Research, ${ }^{3}$ Department of Medical Oncology, City of Hope National Medical Center, Duarte, California.

${ }^{4}$ Department of Biostatistics, Vital Research, Los Angeles, California.
} 
those afflicted and their common comorbidities such as chronic obstructive pulmonary disease. ${ }^{3}$ To deal with this, medical centers must be able to anticipate the types, amounts, and severities of symptoms patients with lung cancers experience in attempts to place necessary resources in arenas that will most benefit these patients. With better efficiency in care of patients with lung cancer, more utility can be had of the almost $\$ 10$ billion spent annually treating these patients. ${ }^{4}$

Little data exist that represents the effect of symptom experience on resource utilization and quality of life in patients with lung cancer. To better depict the range of symptoms in these patients and how that translates to resource utilization, this study sought to retrospectively describe the experience of patients with lung cancer presenting to a cancer center. By doing so, this study may serve as a guide in the development of more effective support services and resource allocation for patients with lung cancer.

\section{METHODS}

After necessary approval from the institutional review board, consecutive patients with a primary diagnosis of lung cancer (small cell and nonsmall cell histologies) treated at a National Cancer Institute (NCI)-designated Comprehensive Cancer Center were retrospectively identified through a computer search of all new patients via a tumor registry over a 1-year period. Of the 125 listed, the first 100 charts were reviewed. The reviews were conducted by three expert clinicians with interreviewer correlation over $95 \%$.

Data gleaned from the retrospective review of the charts included demographic data such as age, gender, race, and ethnicity. Any previous medical history including prior neoplasms and comorbidities was entered into the database. Pulmonary function test results were included. The primary lung histology, stage, previous treatments (surgery, radiation, chemotherapy) and present symptoms were added. Treatment received at City of Hope was next entered. Information pertaining to any hospital admissions such as reason for admission, length of stay, number of ventilator days, and length of intensive care unit stay were entered. The review included each subsequent encounter with a physician, physician extender, or member of supportive care services staff for a period of 6 months after the first encounter at the cancer center. This included the reasons for encounter (e.g., symptom management, postoperative visit, routine visit, chemotherapy), where the encounter took place (e.g., urgent care, clinic, telephone), and whether the en- counter was scheduled or unscheduled. The data were then placed into a database and audited to ensure reliability. Analysis was then undertaken using descriptive statistics. Data files were initially managed in SAS (SAS, Cary, NC) then restructured and analyzed using SPSS for Windows version 12.0 (SPSS, Chicago, IL). Missing data on key static variables were imputed using multiple imputation software.

\section{RESULTS}

Of the 100 charts retrospectively reviewed, 4 patients had small cell and 96 patients had non-small cell lung cancer. Fifty-three of the patients were male. The mean age was 67 years (range, $41-85$ years). Caucasians were the most common $(n=84)$, followed by Asians $(n=13)$, and African Americans $(\mathrm{n}=5)$. Eighty-three percent of the patients presented to City of Hope at diagnosis $11 \%$ with disease persistence, and $6 \%$ patients after disease recurrence Patient staging for the group is shown in Table 1.

Eighteen patients (18\%) had a history of at least one other neoplastic disease; 9 patients had breast cancer; 3 had prostate cancer, 2 had melanoma, 2 cervical, and one each for, colon, gynecology, skin, and thyroid. Nine of these patients with additional cancers presented synchronously with their lung cancers. Four patients had either recurrent or persistent non-lung cancers. Eight patients had no evidence of their prior tumor. Their prior treatments for the other cancers included surgery ( $89 \%$ of patients), radiation (39\%), and chemotherapy (28\%).

Most of the patients (80\%) in the study were previous smokers with a median of 40 pack-years history of smoking (range, 0-150 pack-years). Not surprisingly, $82 \%$ of patients had a median of two comorbidities (range, 0-6). The most common were hypertension (52\%), hypercholesterolemia (27\%), pulmonary disease $(23 \%)$, diabetes $(22 \%)$, heart disease (prior myocardial infarction or angina) (21\%), arthri-

Table 1. Distribution of Stage

\begin{tabular}{lc}
\hline Stage & Frequency $(\mathrm{n}=100)$ \\
\hline IA & 3 \\
IB & 5 \\
IIA & 3 \\
IIB & 3 \\
IIIA & 15 \\
IIIB & 24 \\
IV & 43 \\
Small cell & 4 \\
\hline
\end{tabular}


tis $(20 \%)$, anxiety/depression $(17 \%)$, and neurologic diseases $(12 \%)$.

Only 18 patients were asymptomatic at presentation. The other 82 patients presented with a mean of 1.9 symptoms each. The most common symptoms were pain $(46 \%)$, cough $(44 \%)$, dyspnea $(37 \%)$, fatigue (20\%), gastrointestinal symptoms $(16.5 \%)$, and neurologic symptoms (13\%) (Table 2). Only 53\% of this sample of patients with lung cancer had pulmonary function tests within the 6 months of presentation.

Thirty-nine percent of patients underwent operations. Overwhelmingly, the operation was part of the primary therapy for the tumor (69\%). Ten percent were for locoregional recurrence, $14 \%$ were for symptom management, and $7 \%$ were for distant metastases. Fourteen patients were ASA III while 13 were ASA IV. Fourteen percent were emergent. Lobectomy was the most common procedure (41\%) followed by bronchoscopy/mediastinoscopy (26\%), wedge resection $(21 \%)$, thoracotomy $(13 \%)$, pneumonectomy $(10 \%)$, lymphadenectomy (10\%), and abdominal operation $(10 \%)$. Twenty of the patients undergoing operations required a mean 2.81 day stay in the intensive care unit (range, 1-11 days). Six of these patients required postoperative ventilatory assistance (mean, 2.80 days; range, 1-7 days). Ten patients experienced 16 complications. Pneumothorax, cardiac arrhythmias, and pulmonary dysfunction each occurred in 3 patients. Three patients required reoperation.

Twenty-nine percent of patients underwent some treatment with chemotherapy prior to presenting at City of Hope. Of these, $72 \%$ completed their course. Seventeen patients received radiation prior to presenting at City of Hope, with $94 \%$ of those patients completing their treatment. Upon arriving to City of Hope, $81 \%$ of patients received chemotherapy and $43 \%$ received radiation. Forty-nine percent of patients getting chemotherapy at City of Hope do not finish their designated regimen. This is most often due to tumor progression (39\%) and side effects (38\%). Eighty-six percent of radiation patients finished their treatment.

By its very nature, the retrospective study design offered the opportunity to acquire comprehensive data

Table 2. Symptoms in Patients with Lung Cancer

\begin{tabular}{lc}
\hline Symptom & Frequency \\
\hline Pain & 46 \\
Cough & 44 \\
Dyspnea & 37 \\
Fatigue & 20 \\
Gastrointestinal & 17 \\
Neurologic & 13 \\
\hline
\end{tabular}

Table 3. Support Service Utilization

\begin{tabular}{lc}
\hline Service & Frequency \\
\hline Number of patients with referral & $57(57 \%)$ \\
Nutrition & $40(40 \%)$ \\
Pulmonary rehabilitation & $31(31 \%)$ \\
Physical/occupational therapy & $19(19 \%)$ \\
Social work & $14(14 \%)$ \\
Pain management & $9(9 \%)$ \\
Chaplain & $7(7 \%)$ \\
Psychiatry & $3(3 \%)$ \\
\hline
\end{tabular}

on all admissions and encounters during treatment. Attesting to the predominately outpatient management of patients with lung cancer, only 84 admissions occurred in 57 patients. Thirty-six patients had a single admission, 15 patients had 2 admissions, 5 patients had 3 admissions, and a single patient had 4 admissions during the study period. Most often, patients were admitted to either the surgery service (49\%) or medical oncology (46\%). The reasons for admission were surgery (49\%), symptom management (49\%), and chemotherapy/radiation (2\%). The symptoms most often requiring inpatient management were pain $(43 \%)$, dyspnea $(31 \%)$, nausea (17\%), cough (14\%), and fatigue (12\%). Median length of stay was 7 days (range, 1-17 days). On discharge, $69 \%$ of patients went home independently, $10 \%$ went home with some form of home health, $7 \%$ went to a skilled nursing facility, and $2 \%$ went home with hospice. Seven percent of the patients died in the hospital.

The median number of encounters during the 6 months after presentation was 28 per patient (range, 0-90). Nine percent were unscheduled. The outpatient clinic was the most common venue, accounting for $92 \%$ of encounters, followed by the telephone $(6 \%)$ and urgent care $(3 \%)$. These encounters were most often with the physician (54\%) or physician extender $(43 \%)$. Visits to a supportive care practitioner occurred in $2 \%$ of encounters. The most common departments of encounter were medical oncology $(46 \%)$, radiation oncology (28\%), radiology (6\%), surgery (5\%), and other medical department (4\%). Reasons for encounter included scheduled follow-up visit $(31 \%)$, laboratory evaluations $(30 \%)$, radiation treatment $(20 \%)$, chemotherapy $(17 \%)$, symptom management $(7 \%)$, consultation $(7 \%)$, counseling $(6 \%)$, education $(3 \%)$, routine postoperative visit $(0.4 \%)$, and surgery $(0.3 \%)$. Only $10 \%$ of these encounters were for new problems.

A wide variation in supportive service referrals was seen. A total of 144 referrals in 64 of the 100 patients were made. As shown in Table 3, nutrition was the 
most common (40 referrals), followed by pulmonary rehabilitation (31 referrals), physical and occupational therapy (19 referrals), social work (14 referrals), pain management ( 9 referrals), chaplain (7 referrals), and psychiatry (3 referrals).

Intake records were reviewed to determine what types of home assistance each patient had. In this sample, patients were most often cared for at home by a spouse $(n=61)$, offspring $(n=30)$, friend $(n=6)$, or other relative $(n=3)$. Four patients lived alone and did not have anyone to assist them (Table 4).

\section{DISCUSSION}

The retrospective review of 100 patients seen within a year demonstrated how valuable resources were being allocated and whether they were targeting the aspects most needing attention. This data shows that better resource utilization is possible in treating the quality of life concerns of patients with lung cancer. Although performed at a NCI-designated cancer center, these results show shortcomings in the treatment of patients with lung cancer.

Across each quality of life factor, a wide variation in symptom severity was evident, as some patients experienced symptoms worse than others and reacted to them differently. Regardless of the cause, any decrease in psychosocial function can results in greater distress and lower quality of life. ${ }^{5}$ Fears of being a financial burden, causing familial distress, and an inability to perform home activities were the most affected in the prospective group. Overall uncertainty also greatly affects patients' quality of life. Although the responsibility of all treating disciplines, these concerns were poorly addressed, as was evidenced by the few number of patients seen by social work (14\% of patients), chaplaincy (7\%), or psychiatry (3\%). Evidently, these fears are not without justification, because the vast proportion of these older patients were cared for at home by spouses, most of them of similar advanced age. To address these concerns, specialty referrals such as home

Table 4. Home CARE

\begin{tabular}{lc}
\hline Caregiver & Frequency $(\mathrm{n}=100)$ \\
\hline Spouse & $61(61 \%)$ \\
Children & $30(30 \%)$ \\
Friends & $6(6 \%)$ \\
Other relatives & $3(3 \%)$ \\
Parents & $2(2 \%)$ \\
Other & $2(2 \%)$ \\
Unknown & $3(3 \%)$ \\
Live alone & $4(4 \%)$ \\
\hline
\end{tabular}

health, pulmonary rehabilitation, physical therapy, and occupational therapy could have been utilized to maximize the patient's individual performance status and allay these fears. The undertreatment of these patients and poor regard for their quality of life reiterate results from Sarna et al. ${ }^{6}$ In that study, approximately a third of patients experienced wheezing that required them to stop for breath. Unfortunately, only $18 \%$ of patients were being treated with bronchodilators. The same is seen in the diagnosis and treatment of depression in cancer patients. ${ }^{7,8}$ Clearly, these data show that health care practitioners are inadequately addressing those fears and symptoms most affecting our patients.

Pain was the most common symptom experienced in both cohorts, afflicting $36 \%$ of patients. Yet only $9 \%$ were referred to a pain management service. Without follow up data, this is difficult to interpret, but likely represents underutilization of the pain management service. In fact, pain is known to be extremely common in these patients and, unfortunately, widely undertreated. ${ }^{9-13}$ While there are several possible explanations for this, it is likely advanced age, poor performance status, and high prevalence of other comorbidities and risk factors make these patients' pain difficult and intimidating to treat. ${ }^{14}$ Patients with cancer with pain are among the most difficult to treat, requiring much time and effort on the part of the physicians and the healthcare team. Increasing efficiency and considering the specific symptoms of these patients in office design and execution can significantly improve the quality of palliative care. ${ }^{15}$ By treating most lung cancer patients in oncology offices, efficiencies in care utilized in pain management practices are lost and, most importantly, many patients will likely suffer as their pain is not treated by a specialist.

In this sample, 40 patients were seen by the nutritionist. On further review, these consultations were exclusively in radiation therapy. The reason for this is that the nutritionist is in the radiation oncology department regularly. This shows the importance of building supportive services into health care delivery systems so as to optimize care and efficiencies.

The use of a retrospective cohort in this study showed how resources were being utilized over a 1-year period in a Comprehensive Cancer Center. As with any retrospective study, problems that bias the study may arise. Missing or missed data could have affected our results. However, this was likely minimized by the use of only three reviewers to perform the chart reviews with an interreviewer correlation of over 95\%. Secondly, the full resource use only included that in the Cancer Center. Some patients may have received care in the community rather than solely at the Cancer Center. However, most notes from outside practitioners were included in 
the hospital medical records and were available for inclusion in this study.

Symptom palliation and quality of life are extremely closely related. ${ }^{16,17}$ Thus, in patients with lung cancer it becomes of paramount importance to control their symptoms. Use of limited resourced must address this in the most efficient ways possible. To do so, practitioners must reassess patterns and timing of referrals to supportive care services. Because of the aging population and better noncurative therapies, more patients with cancer will be seen in the near future, taxing local, state, and federal resources. Without better resource utilization and allocation through improvement in efficiencies, a critical shortage of either services or funding will become evident, making amelioration of symptoms and ease of suffering all the more difficult.

This first phase of research served as a descriptive, retrospective review to establish usual care practices for patients with lung cancer at a cancer center. The second phase of this research has now begun and is devoted to the development and pilot testing of a model program for supporting patients with lung cancer. This will occur through the use of a patient and family caregiver interviews to gain subjective perspectives as how to best offer support using a quality of life model encompassing physical, psychological, social, and spiritual well-being. The goal of the interviews, to be conducted individually and in focus groups, is to address needs in lung cancer across a trajectory from initial diagnosis, through treatment and remission, and to recurrence and end of life. While the details of the interventions are to be developed through the interviews, we anticipate the model will likely include aspects of quality of life assessment, symptoms assessment and management, goal setting, patient/family education, and transition to palliative care. This model intervention will be piloted in a small sample with the third phase planned for a major project that would test the lung cancer quality of life intervention in a controlled trial. Our hope is to create a model support program for lung cancer that can be repeated in other settings.

\section{REFERENCES}

1. 〈www.cancer.org/docroot/CRI/content/CRI_2_4_1 $\times$ What_Are_the_Key_Statistics_About_Lung_Cancer_15. asp?sitearea) (Last accessed June 21, 2006).

2. Jemal A, Tiwari RC, Murray T, Ghafoor A, Samuels A, Ward E, Feuer EJ, Thun MJ; American Cancer Society: Cancer statistics, 2004. CA Cancer J Clin 2004;54:8-29.

3. 〈www.seer.cancer.gov/statfacts/html/lungb.html (Last accessed April 3, 2006).
4. Brown ML, Riley GF, Schussler N, Etzioni RD: Estimating health care costs related to cancer treatment from SEERMedicare data. Med Care 2002;40(8 Suppl):IV-104-117.

5. Carlson L, Bultz B. Efficacy and medical cost offset of psychosocial interventions in cancer care: Making the case for economic analyses. Psychooncology 2004;13:837-849.

6. Sarna L, Evangelista K, Tashkin D, et al. Impact of respiratory symptoms and pulmonary function on quality of life and long-term survivors of non-small cell lung cancer. Chest 2004;125:439-445.

7. Patrick DL, Ferketich SL, Frame PS, Padilla G, Holmes C, Brecht ML, Grannis F: National Institutes of Health Stateof-the-Science Conference Statements: Symptom management in cancer: Pain, depression, and fatigue, July 15-17, 2002. J Natl Cancer Inst 2003;95:1110-1117.

8. Stiefel R, Die Trill M, Berney A, Olarte JM, Razavi A: Depression in palliative care: A pragmatic report from the Expert Working Group of the European Association of Palliative Care. Support Care Cancer 2001;9:477-488.

9. Kvale PA, Simoff M, Prakash UB: Palliative care. Chest 2003;123(Suppl 1):284S-311S.

10. Griffin JP, Nelson JE, Koch KA, Neill HB, Ackerman TF, Thompon M, Cole FH, Jr., American College of Chest Physicians: End-of-life care in patients with lung cancer. Chest 2003;123(Suppl 1):312S-331S.

11. Weiss SC, Emanuel LL, Fairclough DL, Emanuel EJ: Understanding the experience of pain in terminally ill patients. Lancet 2001;357:1311-1315.

12. Zhukovsky DS, Gorowski E, Hausdorff J, Napolitano B, Lesser M. et al. Unmet analgesic needs in cancer patients. J Pain Symptom Manage 1995;10:113-119.

13. Di Maioi M, Gridelli C, Gallo C, Manzione L, Brancaccio L, Barbera S, Robbiati SF, Ianniello GP, Ferraù F, Piazza E, Frontini L, Rosetti F, Carrozza F, Bearz A, Spatafora M, Adamo V, Isa L, Iaffaioli RV, Di Salvo E, Perrone F: Prevalence and management of pain in Italian patients with advanced non-small-cell lung cancer. Br J Cancer 2004; 90:2288-2296.

14. Gralla RJ. Quality-of-life considerations in patients with advanced lung cancer: effects of Topotecan on symptom palliation and quality of life. Oncologist 2004;9(suppl 6) 14-24.

15. von Plessen C, Aslaksen A. Improving the quality of palliative care for ambulatory patients with lung cancer. $\mathrm{Br}$ Med J 2005;330:1309-1313.

16. Osoba D, Murray N, Gelmon K, et al. Quality of life, appetite, and weight change in patients receiving dose-intensive chemotherapy. Oncology (Huntingt) 1994;8:61-65.

17. Ruckdeschel JC, Piantadosi S. Quality of life in lung cancer surgical adjuvant trials. Chest 1994;106(suppl 6): 324S-328S.

Address reprint requests to: Betty R. Ferrell, Ph.D. City of Hope National Medical Center 150 East Duarte Road Duarte, CA 91010

E-mail: bferrell@coh.org 